EUROPEAN ORGANIZATION FOR NUCLEAR RESEARCH

ORGANISATION EUROPÉENNE POUR LA RECHERCHE NUCLÉAIRE

CERN-PH-EP/2006-021

CERN-TS-2006-002

27 June 2006

\title{
PREDICTION OF THE THERMAL ANNEALING OF THICK OXIDE METAL-OXIDE-SEMICONDUCTOR DOSIMETERS IRRADIATED IN A HARSH RADIATION ENVIRONMENT
}

\author{
F. Ravotti ${ }^{1}$, M. Glaser ${ }^{1}$, F. Saigné ${ }^{2}$, L. Dusseau ${ }^{2}$, G. Sarrabayrouse ${ }^{3}$ \\ ${ }^{I}$ Technical Support and Physics Department, CERN, Geneva 23, Switzerland \\ ${ }^{2}$ Université Montpellier II, 34095, Montpellier cedex 05, France \\ ${ }^{3}$ LAAS-CNRS, 7 avenue du colonel Roche, 31077, Toulouse cedex 04, France
}

\begin{abstract}
$\underline{\text { Abstract }}$
Radiation-sensing MOSFET transistors produced by the laboratory LAAS-CNRS were exposed to a harsh hadron field that represents the real radiation environment expected at the CERN Large Hadron Collider Experiments. The long-term stability of the transistor's $\mathrm{I}_{\mathrm{ds}}-\mathrm{V}_{\mathrm{gs}}$ characteristic was investigated using the isochronal annealing technique. In this work, devices exposed to high intensity hadron levels $\left(\Phi \geq 10^{12}\right.$ neutrons $\left./ \mathrm{cm}^{2}\right)$ show evidences of displacement damages in the $\mathrm{I}_{\mathrm{ds}}-\mathrm{V}_{\mathrm{gs}}$ annealing behavior. By comparing experimental and simulated results over fourteen months, the isochronal annealing method, originally devoted to oxide trapped charge, is shown to enable prediction of the recovery of silicon bulk defects.
\end{abstract}

Paper submitted to Applied Physics Letters 
At the CERN Large Hadron Collider (LHC) Experiments, Radiation-Sensing MOSFET transistors (RadFETs) ${ }^{1}$ will be widely employed for measuring the Total Ionizing Dose (TID) in the expected mixed electromagnetic and hadronic radiation field ${ }^{2}$. RadFETs operation is based on the $\mathrm{I}_{\mathrm{ds}}-\mathrm{V}_{\mathrm{gs}}$ shift induced by the build-up of positive charge in the gate oxide layer of the transistor $\left(\mathrm{Q}_{\mathrm{ot}}\right)$ and at the oxide-semiconductor interface $\left(\mathrm{Q}_{\mathrm{it}}\right)^{3}$. The degradation of the MOS characteristics reported in Eq. (1) is usually evaluated using the shift of the transistor threshold voltage $\mathrm{V}_{\mathrm{T}}$. For practical reasons, in the case of RadFETs, the measured quantity is a voltage $V_{\text {gs }}$ from Eq. (1) for a given constant $\mathrm{I}_{\mathrm{d}}$. This current, typically $100 \mu \mathrm{A}$, is supplied through the drain-gate terminals, while source and bulk terminals are grounded. In Eq. (1), the constant $\lambda$ only depends on the channel geometry and on the channel carrier mobility $(\mu)$.

$$
I_{d}=\beta \cdot\left(V_{g s}-V_{T}\right)^{2} \cdot\left(1+\lambda V_{d s}\right)
$$

Since the intensity of the radiation field at the LHC Experiments will vary several orders of magnitude, high sensitivity p-MOS transistors, manufactured at the laboratory LAAS-CNRS in France, with a $1.6 \mu \mathrm{m}$ thick gate oxide and sensitivity in the cGy range were investigated at $\mathrm{CERN}^{4}$. The log-log plot of $\Delta \mathrm{V}_{\mathrm{gs}}$ as function of the TID for these devices shows that $\Delta \mathrm{V}_{\mathrm{gs}}$ varies linearly up to 20 Gy. Although it is still possible to measure TID increases for higher doses, $\Delta \mathrm{V}_{\mathrm{gs}}$ becomes strongly sub-linear moving quickly toward saturation after $500 \mathrm{~Gy}$.

The time-recovery evaluation of both $\mathrm{Q}_{\mathrm{ot}}$ and $\mathrm{Q}_{\text {it }}$ trapped charge (i.e. annealing of $\Delta \mathrm{V}_{\mathrm{gs}}$ ) following hadron exposure becomes a crucial parameter to guarantee reliable measurements with RadFETs over the 10-years of LHC operation. Since is not possible to study the annealing of $\Delta \mathrm{V}_{\mathrm{gs}}$ over a time-scale comparable with the LHC operation, the components evaluation is based on the experimental isochronal annealing method originally dedicated to oxide trapped charge ${ }^{5,6}$. This technique consists in emptying sequentially a fraction of the defects during a brief annealing 
period at incremental temperatures. After each annealing step, the effects on $\Delta \mathrm{V}_{\mathrm{gs}}$ are measured at room temperature. The presented annealing studies were performed using a $20^{\circ} \mathrm{C}$ temperature step staircase function and a 360s dwell time. No bias is applied during isochronal and isothermal annealing. From the isochronal annealing curve, characteristics temperatures $\mathrm{T}^{*}$ are extracted, as well as the proportion of defects at a given temperature. $T^{*}$ corresponds to transition temperatures associated to different activation energy levels. Using the activation energy levels calculated from $\mathrm{T}^{*}$, it is then possible to evaluate the annealing of defects at any temperature for any time without performing isothermal annealing experiments.

The irradiation experiments presented in this letter were performed in the secondary radiation environment of the IRRAD1 facility at the CERN-PS accelerator as well as in its primary $23 \mathrm{GeV}$ proton beam. The secondary radiation environment consists of high-energy photons, broad-spectrum neutrons and charged particles in the $\mathrm{GeV}$ energy range produced by the dumping of the primary proton beam into an iron block ${ }^{7}$. At the irradiation positions used for the presented experiments, the contribution of charged particles is always two orders of magnitude lower with respect to the neutrons component; thus only neutron fluences will be quoted in the following.

The dotted curves in Figure 1 plotted with squared and triangular markers represent isochronal annealing behaviors for two devices irradiated in the mixed-hadron field at dose levels of 3 Gy and 10 Gy respectively. In all presented curves, $\Delta \mathrm{V}_{\mathrm{gs}}$ has been normalized to unity and its unannealed fraction is thus used to quantify the annealing process. The two devices show a remarkable stability of $\Delta \mathrm{V}_{\mathrm{gs}}$ that does not start to recover until temperatures exceeding $250^{\circ} \mathrm{C}$. A similar behavior has been measured from a third device exposed to $1 \mathrm{~Gy}$ of pure ionizing gamma rays from Cobalt source (round markers in Figure 1). We can then consider that these first three 
measured behaviors are mainly due to the $\mathrm{Q}_{\mathrm{ot}}$ and $\mathrm{Q}_{\mathrm{it}}$. Applying the isochronal method on these data sets it is possible to estimate a room temperature recovery of $\Delta \mathrm{V}_{\mathrm{gs}}$ less than $1 \%$ over $10^{4}$ hours (taken $1 \times 10^{7} \mathrm{sec}^{-1}$ as factor of thermal acceleration ${ }^{8}$ ). This prediction is in excellent agreement with experimental data presented in references ${ }^{9,10}$ for the same devices exposed to tens of Gy in a pure ionizing radiation environment. The maximum neutron fluence that hits the device exposed to 10 Gy does not exceed $1.5 \times 10^{11} \mathrm{~cm}^{-2}$. In agreement with a previous work ${ }^{11}$, this level of neutron irradiation is not expected to induce any measurable changes in the $\mathrm{I}_{\mathrm{ds}}-\mathrm{V}_{\mathrm{gs}}$ characteristics.

The curve plotted with diamond markers in Figure 1 shows instead the isochronal annealing behavior of a fourth MOSFET irradiated up to a TID of $854 \mathrm{~Gy}$. In this case the device has been exposed in parallel to a high neutron fluence of about $2 \times 10^{13} \mathrm{~cm}^{-2}$. For this sample the $\Delta \mathrm{V}_{\mathrm{gs}}$ recovers about $50 \%$ at a temperature of $250^{\circ} \mathrm{C}$. Clearly at this irradiation level, an overlapping phenomenon that recovers in the presented data must be taken into account. We can consider that, for such neutron fluence, the number of displacement damages is high enough to induce an $\mathrm{I}_{\mathrm{ds}}-\mathrm{V}_{\mathrm{gs}}$ modification: the observed annealing can then be assumed to correspond to displacement damages recovery.

Atomic displacement ${ }^{12}$ in the bulk semiconductor layer can be responsible for the degradation of the transistor channel mobility ${ }^{13}$ that in turn may increase the MOS channel resistance $^{14}$. Via the parameter $\lambda$ the bulk damage can thus degrade the dosimetric signal measured through Eq. (1). Therefore, in order to assess whether the additional degradation in the $\Delta \mathrm{V}_{\mathrm{gs}}$ recovery can be related to the occurrence of displacement damage, a second series of devices was exposed to the pure $23 \mathrm{GeV}$ proton beam of the IRRAD1 facility. The annealing measurements related to this second irradiation test are plotted with filled markers in Figure 1. 
From these last data sets, it is possible to identify a common series of transition temperatures ${ }^{15}$ (inflection point of the experimental isochronal annealing curve) $\mathrm{T}_{1}{ }^{*}=130^{\circ} \mathrm{C}, \mathrm{T}_{2}{ }^{*}=180^{\circ} \mathrm{C}$ and $\mathrm{T}_{3}{ }^{*}=280^{\circ} \mathrm{C}$ that may correspond to the temperatures at which the different types of radiationinduced defects spontaneously dissociate in silicon ${ }^{16}$. According to ref. ${ }^{17}$ these $\mathrm{T}^{*}$ may indicate the recovery of radiation-induced centers formed by vacancy defects (V) in the silicon lattice with impurities atoms or with other vacancies. To verify the above hypothesis, the bulk damage characteristics extracted from the proton isochronal annealing curves can be used now to predict the isothermal annealing behavior of $\Delta \mathrm{V}_{\mathrm{gs}}$ for the heavy irradiated devices. $\Delta \mathrm{V}_{\mathrm{gs}}$, normalized to its initial value $\Delta \mathrm{V}_{\mathrm{gs}}(0)$, is so computed taking into account that the time-dependence of the defects dissociation is described by a first order process ${ }^{18}$ :

$$
\begin{aligned}
& \frac{\Delta V_{g s}(t)}{\Delta V_{g s}(0)}=\sum_{i=1}^{3} B_{i} \cdot \exp \left(-\sigma_{i} t\right) \\
& \sigma_{i}=A_{i} \cdot \exp \left(-\frac{E_{A, i}}{k_{b} T}\right)
\end{aligned}
$$

where: $t \equiv$ annealing time, $\sigma_{\mathrm{i}} \equiv$ probability per unit time for the defect $i$ to dissociate $\left(\mathrm{s}^{-1}\right)$ and $\mathrm{B}_{\mathrm{i}} \equiv$ fraction of $\Delta \mathrm{V}_{\mathrm{gs}}$ that recovers with the dissociation of defect $i$ (experimental parameter from the curves of Figure 1). The temperature dependence of $\sigma_{\mathrm{i}}$ is given by an Arrhenius relation where $\mathrm{A}_{i}$ $\equiv$ factor of thermal acceleration of defect $i\left(\mathrm{~s}^{-1}\right), E_{A, i} \equiv$ activation energy for dissociation of defect $i(\mathrm{eV}), k_{b} \equiv$ Boltzmann's constant and $\mathrm{T} \equiv$ annealing temperature $(\mathrm{K})$. According to ${ }^{17,18,19}$ the characteristic temperatures $\mathrm{T}_{1}{ }^{*}, \mathrm{~T}_{2}{ }^{*}$ and $\mathrm{T}_{3}{ }^{*}$ correspond thus to the recovery of the V-P $\left(\mathrm{A}_{1}=\right.$ $\left.7 \times 10^{8} \mathrm{~s}^{-1} \mathrm{E}_{\mathrm{A}, 1}=0.94 \mathrm{eV}, \mathrm{B}_{1}=0.25\right), \mathrm{V}-\mathrm{Sb}\left(\mathrm{A}_{2}=1 \times 10^{10} \mathrm{~s}^{-1} \mathrm{E}_{\mathrm{A}, 2}=1.28 \mathrm{eV}, \mathrm{B}_{2}=0.25\right)$ and $\mathrm{V}-\mathrm{V}\left(\mathrm{A}_{3}\right.$ $\left.=1 \times 10^{9} \mathrm{~s}^{-1} \mathrm{E}_{\mathrm{A}, 3}=1.47 \mathrm{eV}, \mathrm{B}_{3}=0.5\right)$ centers respectively.

Figure 2 shows finally the comparison between the curve from Eq. (2) and the experimental annealing, recorded over 14 months, for three MOS transistors independently 
irradiated in the same IRRAD1 secondary radiation field. The three devices where exposed to different TIDs and to high neutron fluences ranging from $4.5 \times 10^{12} \mathrm{~cm}^{-2}$ to $2 \times 10^{13} \mathrm{~cm}^{-2}$. It has to be noted that the calculation with Eq. (2) takes into account the real temperature profile recorded during the measurement of the data. We are then able to follow the thermal annealing of the defects at any time of the experiment. The prediction, evaluated on the real temperature profile (see secondary y-axis of Figure 2), fits the experimental data better than $1 \%$ up to about $3 \times 10^{3}$ hours. The accuracy of the calculation is still very good (4-5\%) after fourteen months.

In this work we report that the annealing behavior of RadFET dosimeters irradiated in a harsh radiation field may show a component due to the recover of radiation-induced displacement damages in the n-type silicon bulk of the transistor. This appears when the hadron component of the mixed radiation field exceeds some $10^{12} \mathrm{~cm}^{-2}$. It can be still pointed out that the measurement of the "real" $\Delta V_{T}$ of the transistor will be probably immune to this effect due to its independence in the transistor's channel mobility degradation. The isochronal annealing was used here to evaluate the displacement damage annealing and then the long time behavior of the RadFET dosimeters. The comparison between the annealing prediction and a set of experimental data recorded over 14 months is found to be very accurate. It is then shown that defects induced by harsh radiation environment characteristic of CERN-LHC experiments can be evaluated using the isochronal annealing method. Although bulk damage effects can induce an additional error in the $\Delta \mathrm{V}_{\mathrm{gs}}$ measurement, it is found to be as low as $8 \%$ over 14 months of thermal annealing for absorbed doses up to the kGy range, proving that the tested LAAS devices fully comply with the LHC stability requirements even under extremely high radiation levels.

The authors would like to thank the reviewers and M. Moll from CERN for their useful comments and suggestions. 
${ }^{1}$ A.G. Holmes-Siedle, L. Adams, Rad. Phys. and Chem. 28 (2), pp. 235-244, 1986.

${ }^{2}$ F. Ravotti, M. Glaser, M. Moll, K. Idri, J-R. Vaillé, H. Prevost and L. Dusseau, IEEE Trans. Nucl. Sci, vol. 51 (6), pp. 3642-3648, 2004.

${ }^{3}$ G. Sarrabayrouse, F. Gessinn, Radioprotection, vol. 29 (4), pp. 557-572, 1994.

${ }^{4}$ G. Sarrabayrouse, V. Polischuk, Rad. Phys. and Chem. 61, pp. 511-513, 2001.

${ }^{5}$ F. Saigné, L. Dusseau, J. Fesquet, J. Gasiot, R. Ecoffet, J. P. David, R. D. Schrimpf, and K. F. Galloway, IEEE Trans. Nucl. Sci., vol. 44, pp. 2001-2006, 1997.

${ }^{6}$ F. Saigné, L. Dusseau, J. Fesquet, J. Gasiot, R. Ecoffet, R.D. Schrimpf, K. F. Galloway, IEEE Trans. Nucl. Sci., vol. 48 (6), pp. $2170-2173,2001$.

${ }^{7}$ M. Glaser, L. Durieu, F. Lemeilleur, M. Tavlet, C. Leroy, P. Roy, Nucl. Inst and Meth, A426, pp. $72-77,1999$.

${ }^{8}$ F. Saigné, L. Dusseau, L. Albert, J. Fesquet, J. Gasiot, J. P. David R. Ecoffet, R.D. Schrimpf, K.F. Galloway, J. Appl. Phys., vol. 82, pp. 4102-4107, 1997.

${ }^{9}$ F. Gessinn, G. Sarrabayrouse, Mat. Res. Soc. Symp., 302, pp. 555-560, 1993.

${ }^{10}$ N. Kumurdjian, G. Sarrabayrouse, Rad. Prot. Dos., 61 (1-3), pp. 19-24, 1995.

${ }^{11}$ V.N. Bhoraskar, AIP Conf. Proc., 392, pp. 1025-1028, 1997.

${ }^{12}$ G. Lindström, M. Moll, E. Fretwurst, Nucl. Instr. and Meth., A426, pp. 1-15, 1999.

${ }^{13}$ W.G. Oldham, E.T. Gaw, IEEE Trans. Nucl. Sci., vol. 21 (6), pp. $124-129,1974$.

${ }^{14}$ A. Balasinski, T-P. Ma, IEEE Trans. Nucl. Sci., vol. 39, pp. 1998 -2003, 1992.

${ }^{15}$ L. Dusseau, T. Randolph, R.D. Schrimpf, K. F. Galloway, F. Saigné, J. Fesquet, J. Gasiot, R. Ecoffet, J. Appl. Phys., vol. 81, pp. 2437-2441, 1997.

${ }^{16}$ A.G. Holmes-Siedle, L. Adams, $2^{\text {nd }}$ ed., Oxford University Press, Oxford, 2002.

${ }^{17}$ J. C. Corbett, J. Bourgoin, J. H. Crawford and L. M. Slifkin: Plenum, New York, 1975. 
${ }^{18}$ M. Moll, Ph. D. Thesis, Hamburg University, 1999.

${ }^{19}$ L. Elkin, D. D. Watkins, Physical Review, vol. 174 (30), 1968. 


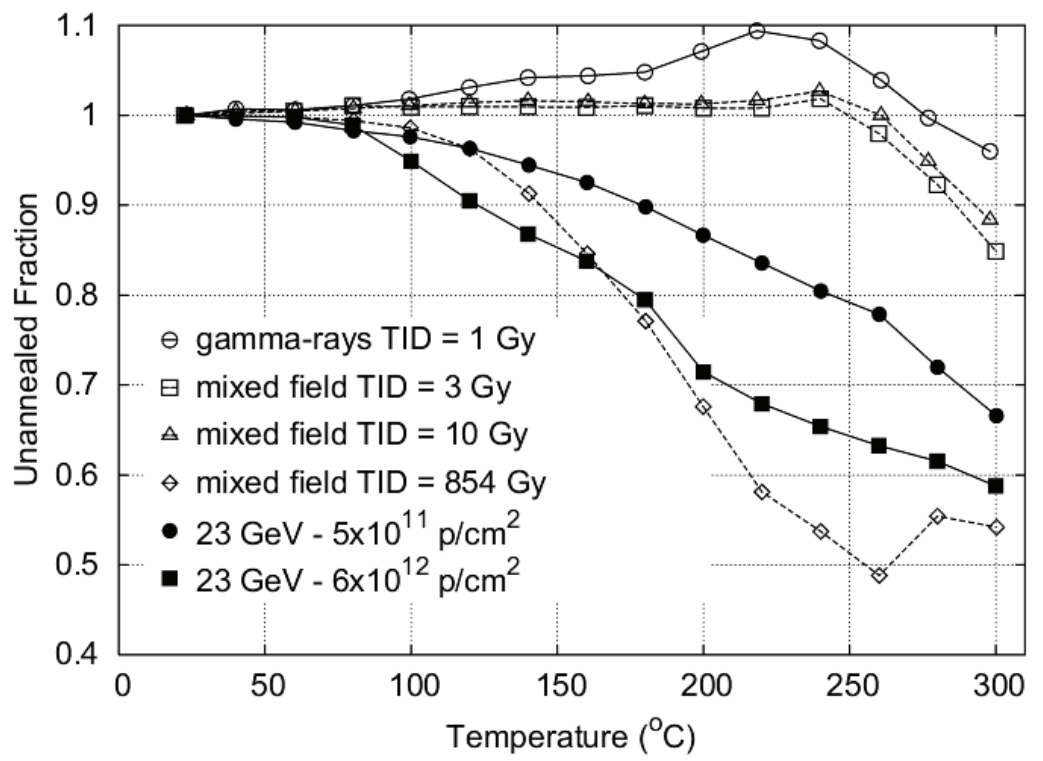

Figure 1: Experimental Isochronal annealing curves for LAAS MOSFETs irradiated in mixed-hadron field (empty markers) and in $23 \mathrm{GeV}$ protons (filled markers). The round markers-continuous line curve shows the isochronal annealing behavior of a device that was irradiated with gamma-rays.

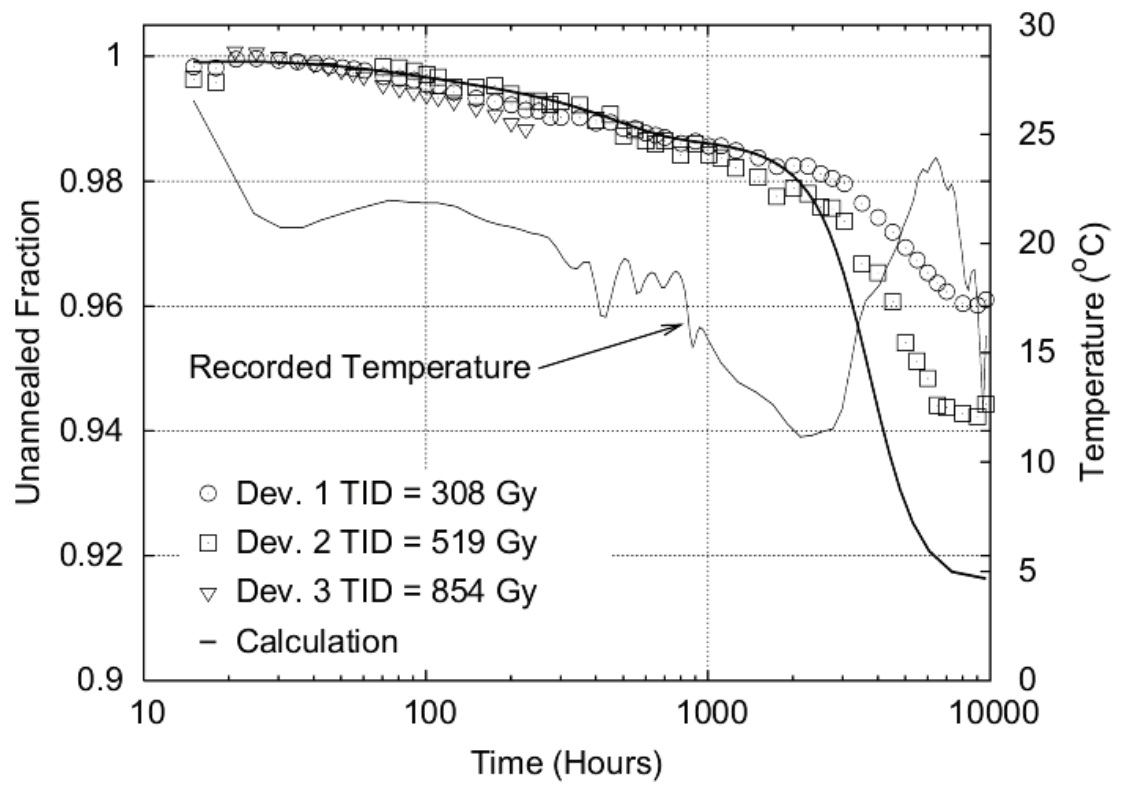

Figure 2: Experimental isothermal annealing curves (different markers) for LAAS devices measured following irradiation in the mixed-hadron field at CERN. The light solid lines represent the temperature recorded during the isothermal measurements, while the heavy line show the results of the isothermal annealing calculation based on the recover of silicon radiation-induced defects taking into account the real temperature profile during the fourteen months experiment. 